\title{
Study on Application of Virtual Reality Technology in the Display of Space Design
}

\author{
Luxin $\mathrm{Ye}^{1}$, Jiayao $\mathrm{Wang}^{1}$ and Jibin Zhang ${ }^{1}$ \\ ${ }^{1}$ Jilin Animation Institute, Changchun, Jilin
}

Keyword: Virtual reality technology; Display of space design; Technology application

\begin{abstract}
Nowadays with increasing economic progress and development of science and technology, human society has entered the digital media era and Virtual reality technology virtual reality is making the leap into everyday life and widely used in all walks of life. The paper is based on the display of space design and develops from many aspects of the development and features and practical applications of virtual reality technology, the specific application of virtual reality technology in modern display space design has been further proposed and Meanwhile make a prediction for the trend of the application development in the future.
\end{abstract}

\section{Definition and Development Demand of Display of Space Design}

With the development of social civilization, display of space design presents historical staging and diversity of the times. The display of modern space design needs to create a multi-dimensional physical space or virtual space through new technologies such as light and electricity in order to mobilize your various senses and experience this time and spatial art. The display design requires not only professional knowledge, but also a variety of disciplines including psychology, aesthetics, ergonomics, communication, economics and sociology. The display of space design is a combination of art and technology and highly integrated art skills presented. Along with the technological revolution,the display of design changes dramatically from within. As a result of the combination of modern scientific and technological achievements and cultural stage, it has great social values. Various categories of museums and galleries act as the function of cultural protection and historical inheritance and also has the function of extending education in improving national quality. Commercial display of space is the process of products transaction and economic circulation of human economy and society. The display of space design should not only have artistic and cultural creative activities, but also shows the applications of scientific and technological achievements. With the development of science and technology, the display of modern space design constantly integrates the new media technology, shows the high technology level and brings the harmonious and beautiful space art experience to the public life.

\section{The Development and Features of Virtual Reality Technology}

The Development of Virtual Reality Technology. The virtual reality technology stems from "sensing cinema", which was invented by Hollywood's Morton Heilich last century. The term "virtual reality", coined by American company Galomlille, was widely followed by the U.S. government and applied to military and aerospace programs. In our country, virtual reality technology started late, but it has been applied to various fields and made breakthrough progress. Virtual reality technology is a comprehensive information technology based on computer graphics, computer simulation, multimedia technology and sensor technology. It simulates real environment using three dimensional of computer simulation and generates real-time dynamic three-dimensional multi-sensory virtual environment. The users influence each other through the sensing devices and interaction of objects in the virtual environment.

Features of Virtual Reality Technology. In recent years, virtual reality technology is appearing in people's life through various forms, especially in the design and application of display space. Virtual reality technology mainly includes three aspects: interactive feature, and imagination feature. 
Interactivity feature. Interactivity mainly refers to the behavior that participants can conduct on the objects in the virtual environment of the computer, which can be fed back to the machine, showing obvious interactivity and real time.

Immersive feature.Immersion mainly refers to that the users are able to put themselves in the virtual world once they enter the virtual environment. They can experience virtual behaviors and sports and feedback activities like real world through sight, hearing, touch, smell and many other perception. The users can end up immersing themselves into it just like the real world.

Imagination feature. Imagination mainly refers to how to reflect the feature of the user's imagination and creativity in VR technology。 The users not just passively accept the preset program information, but rather reflect the operator's subjective initiative, think about and imagine a the future changes of movement according to the changes of running state of system and then promote the deepening, extension of the concept and generate new ideas, actively seek to explore the unknown information, conduct more imagination and creation in the virtual space, involved in forming new virtual activities and dynamic generation. Thus, the virtual reality shows how to reflect the creative thinking of users.

\section{Application of Virtual Reality Technology in the Display of Space Design}

The greatest feature of the display of design space is the strong mobility, the dynamic, serialized and rhythmic display form in space design. Display space requires careful design to create a novel and unique artistic space and achieve the goal of short time and effect in order to achieve the artistic tension of outstanding exhibits. Thus the display of space design also uses its own unique technique to draw the audience to participate intuitively and vividly and make the audience participate personally, to reach the feeling of immersive situation, and thereby understand the exhibits information more deeply. First of all, the basic principles to be followed is determined by the nature of the space and the human factors. People in the display space are in the state of visiting the movement, experience in the movement and gain the final space feelings. This requires that display space must be based on which and get the audience involved in the display activities, completely and economically with the most reasonable method to arrange the audience to streamline without or less repeated routes as much as possible and especially without being repeated in the key area of the display area. In the processing of space, it can be as fluent as music's melody, and the cadence is distinct, so that the whole design is logical. While satisfying the function, let people feel the charm of space change and the infinite interest of design.

Application of Virtual Reality Technology in Display Space Design. In recent years, virtual reality technology has been applied more in the display of space design. The Chinese pavilion has become the largest pavilion country in the world in recent years. But as merchandises get faster and faster, the pavilions require a lot of manpower and a long wait for planning time, as well as a short display of time and a more restrictive geographical limit. So many businesses found that the combination of various advantages of the internet and various advantages of virtual reality display design can bring new vitality to the pavilion, but this pavilion is not entity but a virtual exhibition hall.

Core Application Technology of Virtual Reality Technology in Display of Space Design. First, 3d modeling builds virtual display space. The design principle of virtual reality in $3 \mathrm{~d}$ modeling mainly focuses on strengthening the principle of "commodity description", "display tool" and "lighting" so as to demonstrate the digital virtualization of design as the technical means to strengthen the commodity. In the design, graphic display methods on the basis of the original renderings and posters are transformed technically, from a design principle with main focus on two-dimensional space to $3 \mathrm{~d}$ virtual space. In the original design way what needs to be displayed is restored to 3D model using 3-dimention and the realistic scene is simulated with virtual lighting. After restoring the real scene using virtual means and the basic design principle of the display design is applied to the virtual reality display environment the virtual scene will be full of design elements, close to human and full of life sense . For example, in a virtual mall environment, it is redesigned using the design plan of the display design making the experiencers feel as if they are in the real world and identify commodity information fully. 
On the design steps, scenes are produced mainly through the fine interpretation, scene design drawings of specific data or for existing stores 3-d mapping, and then through the software modeling, texturing, shading and lighting and other technical means. 3d reconstruction of scenarios that will require virtualization is a complex and elaborate technical project.

In the design stage, designers need to understand the type of scene, and have the full understanding of the culture to be spread and commodity information. The virtual display environment focuses more on the communication of the first message.

Secondly, restoring and optimizing the material, color, and lighting atmosphere in the real scenes and revising and optimizing the defects in the real scenes and then highlighting the theme that the virtual display environment displays. Finally, it really displays the combination of design and virtual reality, and provides technical means for the excellent display platform of commodities.

Thirdly, the implementation of design principle of the panoramic virtual display space design is to use the combination of 360-degree panoramic photos to reconstruct the real environment mainly with a fixed angle. What display design in the panoramic environment of virtual reality mainly designs is the coordination of the overall environment and a kind of coordination and unity of the overall sense of color and light feeling. Whether or not there are many real-life objects in the virtual panorama environment that fill the virtual environment, making visitors feel like they are in a real show environment.

In the virtual environment, sometimes some advantages of the virtual space need to be highlighted, such as the narrow road congestion in real stores and virtual environment can widen the roads according to the demand, optimize the road feeling, then the distance of each shop is designed to best visual feeling, more suitable for virtual walking interaction. Such a design process is not isolated from the reality panorama environment, and its display advantages of design of virtual environment is also presented most.

Virtual Reality Technology Achieves the Perfect Unification of Art and Technology in Display Space Design. In the early stage of the development of virtual reality technology, due to the imperfections of the technological process, many flaws were presented, which also reflected some imperfections and even the opposite of art and technology.But with the development of technology and the improvement of process, it is more of perfect union nowadays.

In order to achieve the ultimate goal of presentation, people often use technical updates to constantly mobilize new participative and initiative. Virtual display of space design and information technology can also provide a new platform for artists of course. Artists use visual virtual reality to create more ideas and imagination. Meanwhile, art adds more "color" to the technology. When people continue to explore aesthetic culture and artistic attainments, they feel the unique charm of art and unique perspective, vivid and interesting ways which make people no longer feel bored and enrich people's life and arouse people's interest.Some kind of improvement on things that are already loved bring people different feelings, diversified forms of expression. So far, we cannot affirm the multiple effects of art on virtual reality technology, but there is no doubt about the value of art and emphasis of aesthetic demand. It accelerates the integration of art and technology. Only by combining technology and art and mutual promotion can the artistic charm and high-end technical level of the virtual display design be displayed. From the perspective of sensory, color, structure, atmosphere and so on, the optimization of demonstration effect is achieved, instead of simply relying on the technology or the single aspect of art to attract users to participate.

\section{Future Development Trend of Virtual Reality Technology in Display Space Design}

Audi's VR selection service is a future model of virtual reality technology in display space. The hardware of implementation of the technology is Oculus Rift which uses a head-mounted display. When a user wears it, the visual part of the user senses is placed in virtual space scene. It can not only provide very real image simulation, but also can make the users not only see the environment in the car but also listen to the simulated audio system in the car through the restoration of sound, so as to bring more real user experience. Users can achieve the same thing as sitting in a real car through it and also set up different 
interior decoration, color and vehicle equipment, which greatly improves the efficiency of the user's selection. Technology and art, creation and innovations of new things, and the progress of art promote the emergence of new technologies. Display of space design in our country started relatively later and is still not perfect compared with foreign virtual display area, but from the research production of display design industries in recent years, virtual reality technology has a broad development prospect in the field of display design. Along with the social development and progress, the increasing exchanges between different countries provide a good communication environment for the new media display design industry in our country. More and more new ideas, new technology provide convenience for designers opening new art area. The use of virtual reality technology has led to the development of space design in a more humanized direction. It provides interaction between people and information, and really emphasizes the participation and dominance of humans.

\section{References}

[1] N.Shan and Q.Gong, 2014, display design Chongqing: Southwestern Normal University press.

[2] Z.H.GENG, 2009, Display design in the digital age. Beijing: China Water Conservancy and Hydropower Press.

[3] X.L.Yang, 2015.The application of virtual reality technology in new media exhibition design. Artistic Discovery 54-57.

[4] F.Huang and L.Yu, 2008, A brief introduction to application and technology of virtual reality. Journal of Changchun Institute of Education (4):50-51.

[5] T.Q.Cao,2010, New media experiment and art design. Changsha: Hunan Fine Arts Publishing House. 74.

[6] G.Q.Zhang,2015, The discussion of virtual reality technology. Science and Technology information (30).

[7] M.J.Zhang,2002, virtual reality system. Beijing: Beijing Science Press. 6.

[8] X.Fang et al 2014, digital art of design. Wuhan: Wuhan University of Technology press. 91.

[9] W.Zhang, et al 2016, Research on innovative methods of multimedia interactive design. Journal of Nanjing Arts Institute.

[10]X.T.Lu, 2013, Analysis of the application of virtual reality technology in modern display art. Digital fashion (New Visual Art). 